Supporting Information

Nanostructure of Poly( $\mathrm{N}$-isopropylacrylamide) Brush at the Air/Water Interface and its Responsivity to Temperature and Salt

Hideki Matsuoka* and Kyohei Uda

Department of Polymer Chemistry, Kyoto University, Kyoto 615-8510, Japan

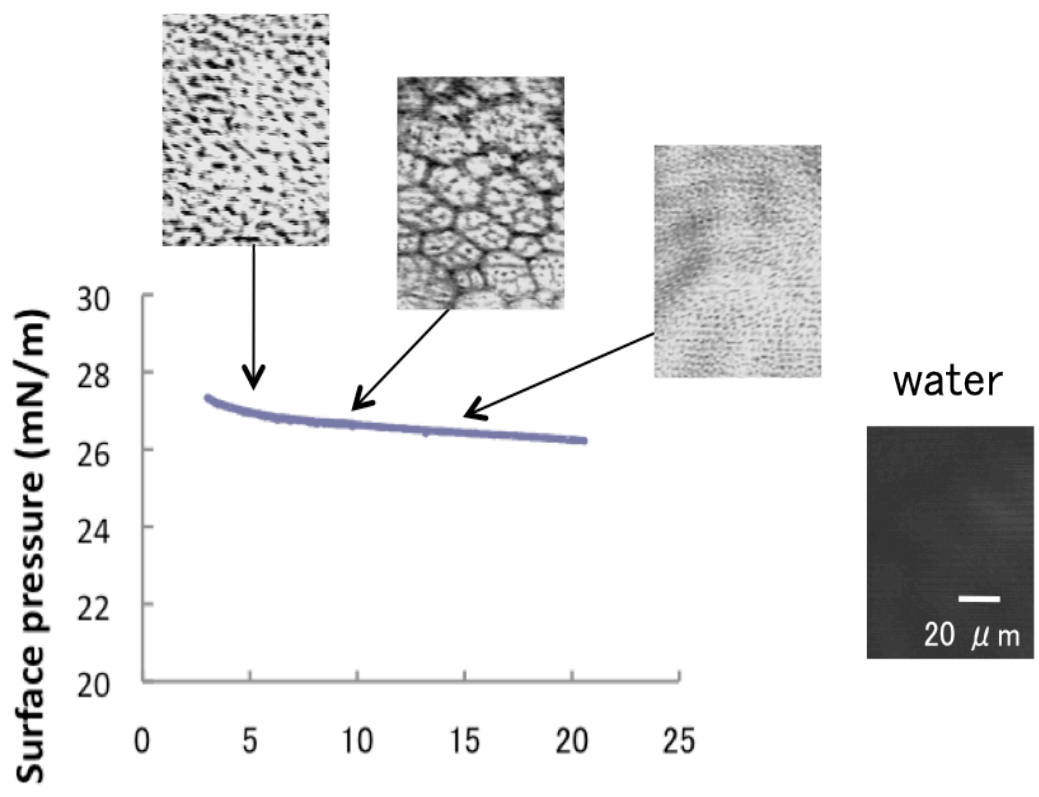

Area $\left(\mathrm{nm}^{2} /\right.$ molecule)

Figure S1 BAM images of $n \mathrm{BA}_{228}$ and $\mathrm{NIPAm}_{79}$ blend monolayer on the water surface at $25^{\circ} \mathrm{C}$.

Mixing ratio is $1: 1$ in monomer molar ratio.
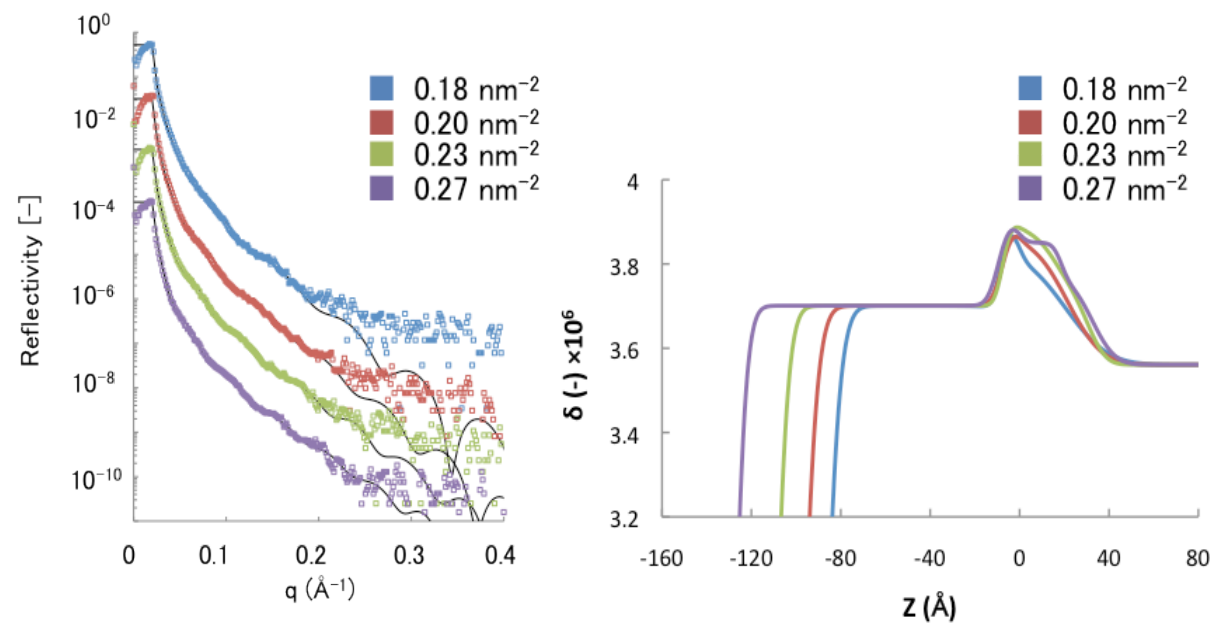

Figure S2 XR profiles (left) and density profiles (right) of $n \mathrm{BA}_{228}-b-\mathrm{NIPAm}_{76}$ monolayer on the water surface at $30^{\circ} \mathrm{C}$ 

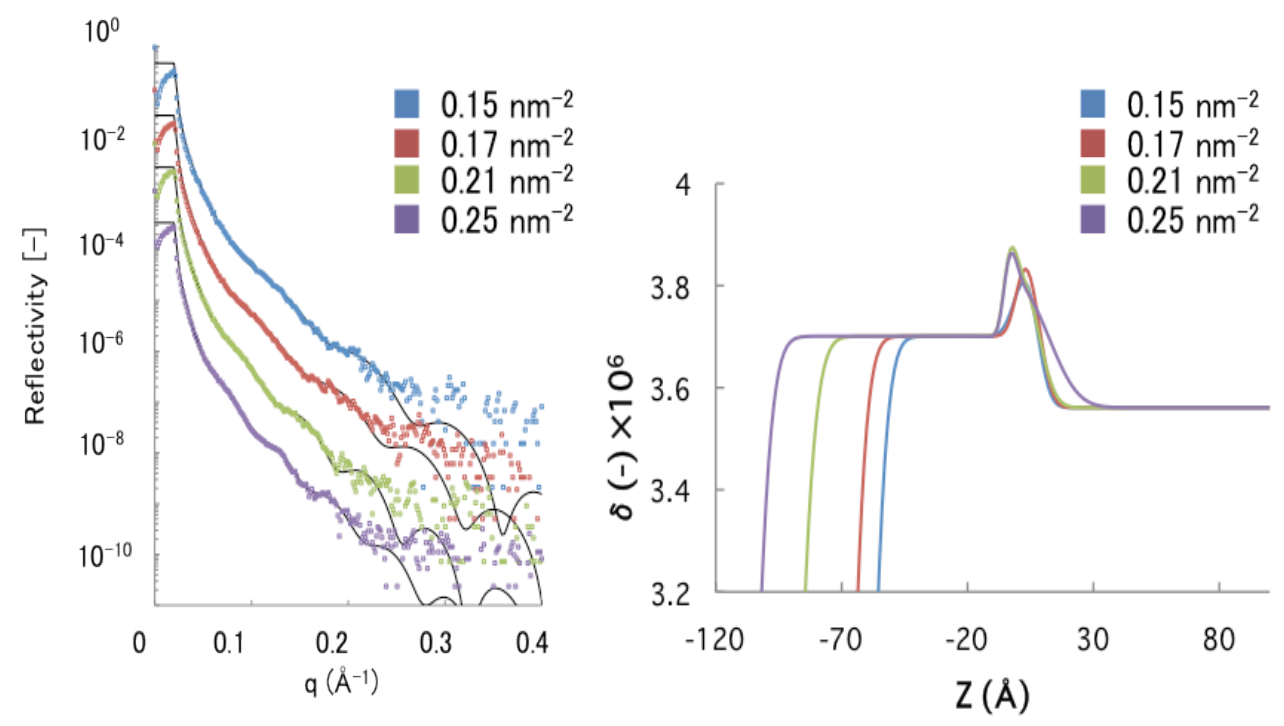

Figure S3 XR profiles (left) and density profiles (right) of $n \mathrm{BA}_{205}-b$-NIPAm 29 monolayer on the water surface at $30^{\circ} \mathrm{C}$.
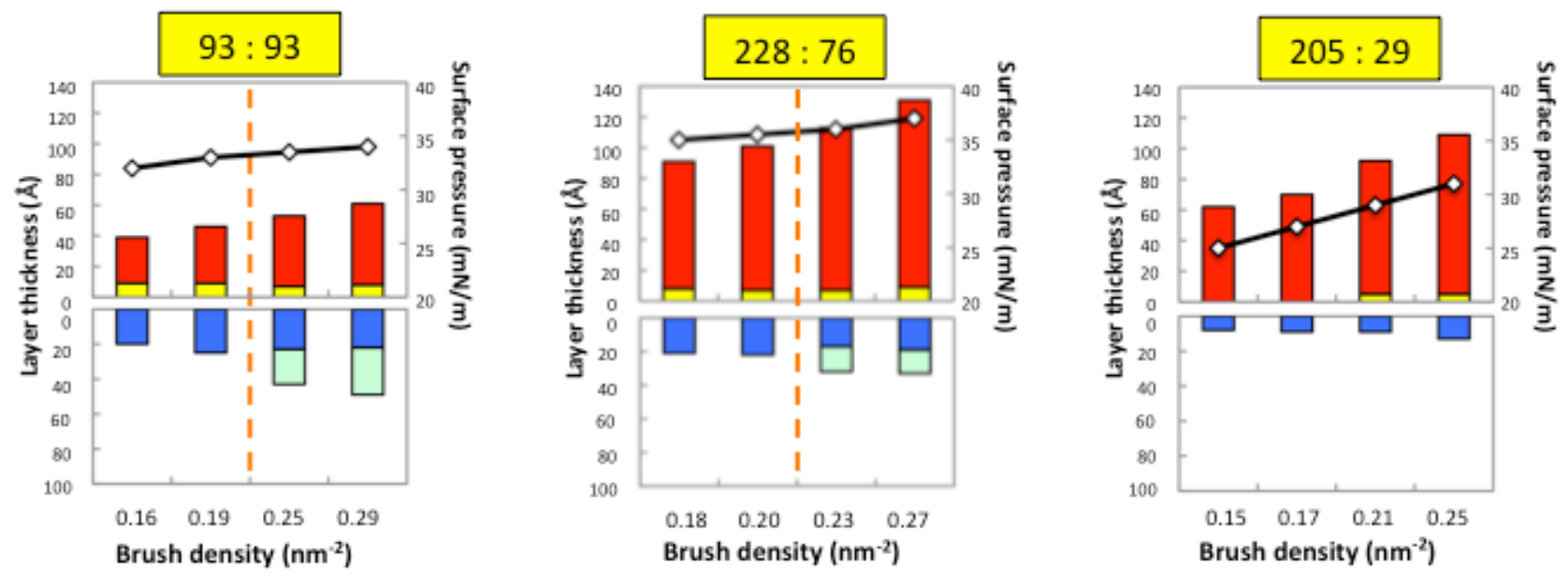

Figure S4. Layer thickness of $(\mathrm{nBA})_{\mathrm{m}}-\mathrm{b}-(\mathrm{NIPAm})_{\mathrm{n}}$ monolayer on water at $30^{\circ} \mathrm{C}$. $m: n=93: 93,228: 76,205: 29$.

口 : hydrophobic layer (nBA) $\square$ : hydrophobic layer (NIPAm) $\square$ : carpet layer $\square$ : brush layer 


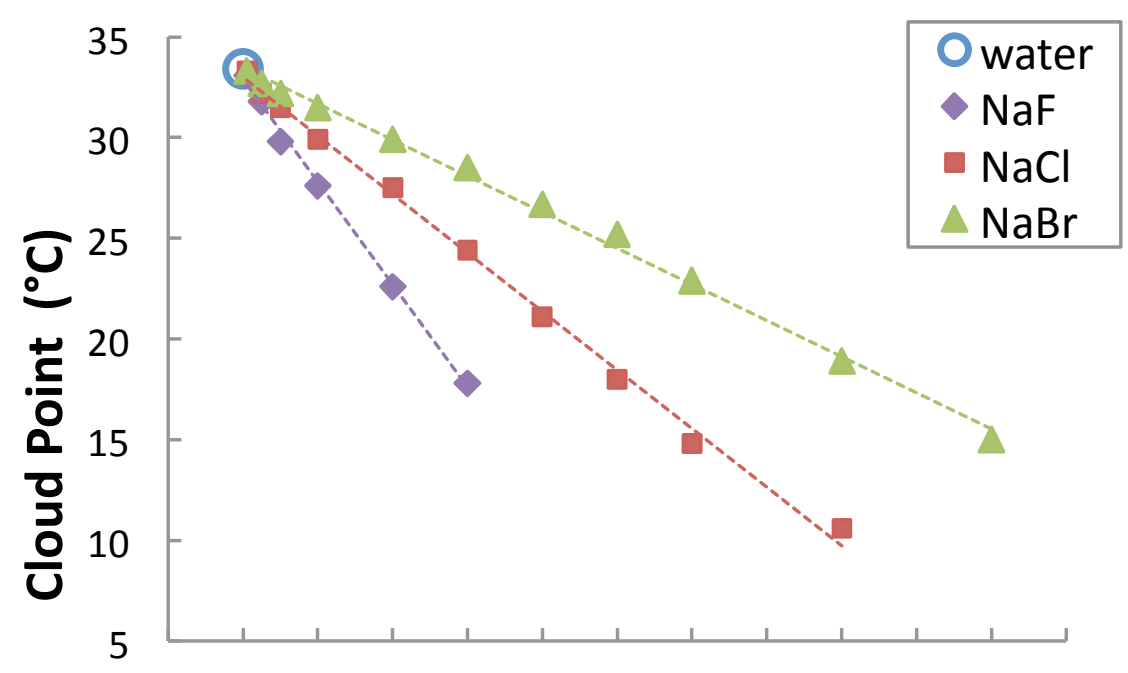

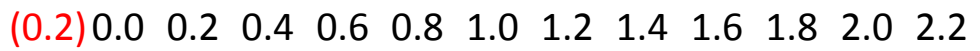

\section{Salt concentration (M)}

Figure S5 Effect of sodium salt concentration on cloud point of PNIPAm aqueous solution. [PNIPAm] $=1 \mathrm{mg} / \mathrm{ml}$, D.P. $=123$

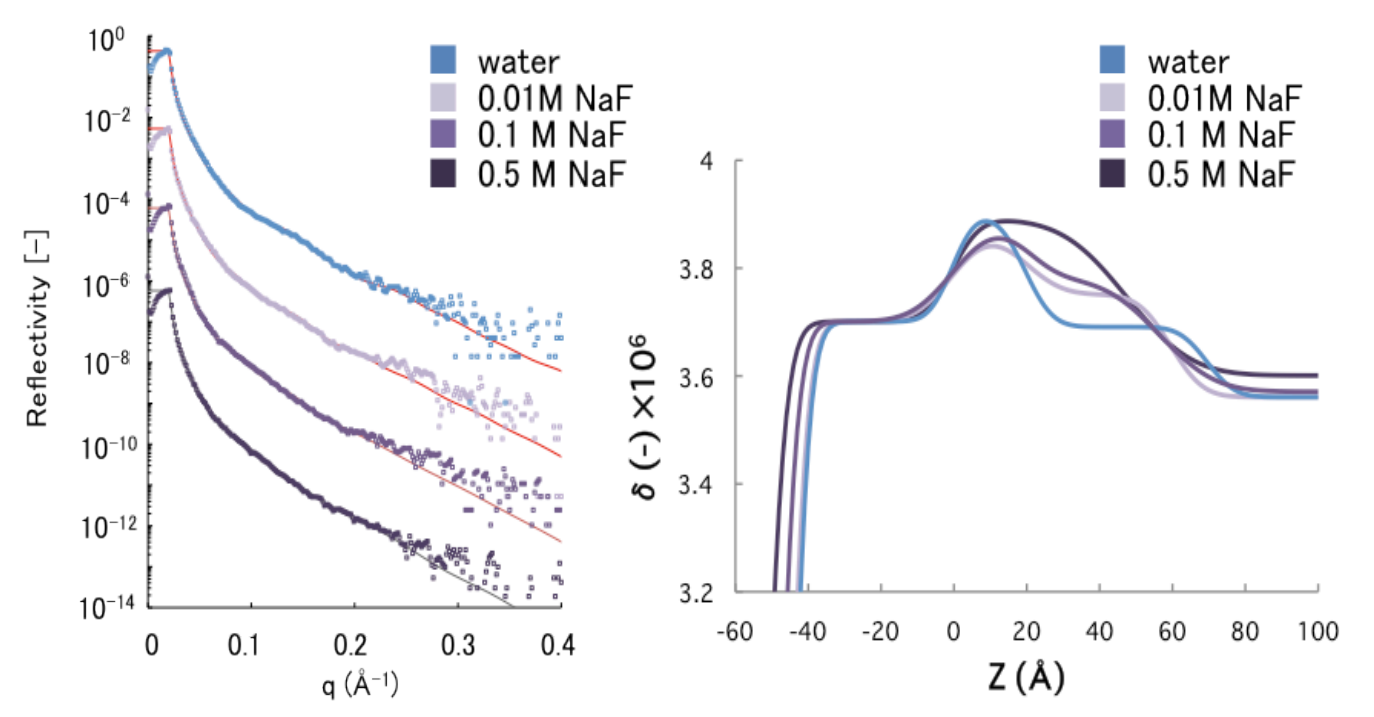

Figure S6 XR profiles (left) and density profiles (right) of $n \mathrm{BA}_{93}-b-\mathrm{NIPAm}_{93}$ monolayer on NaF aq. at $25^{\circ} \mathrm{C}$. $A=3.7-$ $4.0 \mathrm{~nm}^{2}$ 


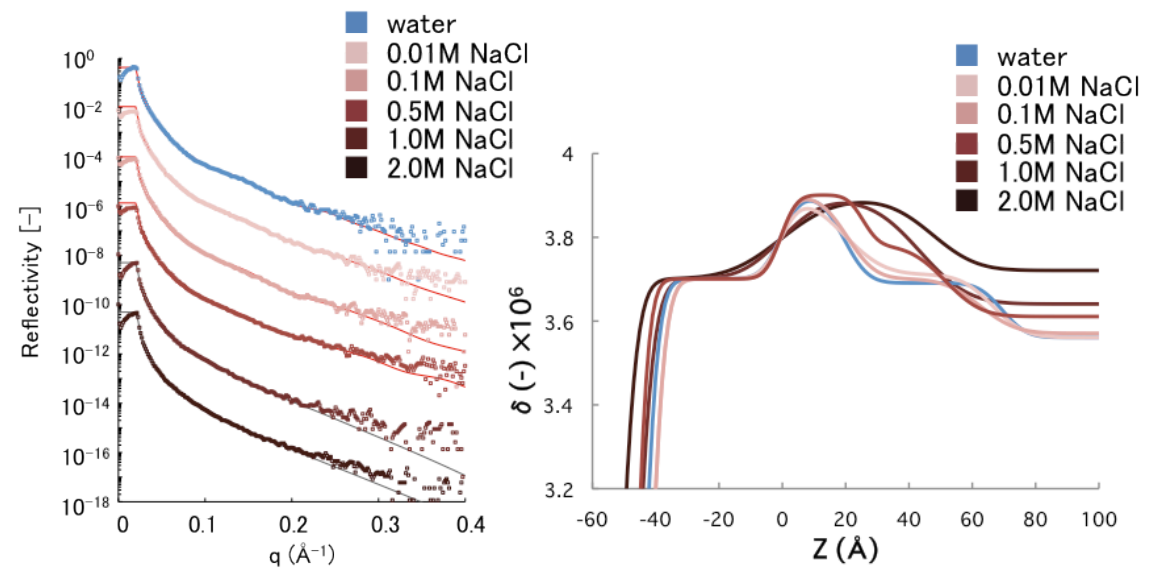

Figure S7 XR profiles (left) and density profiles (right) of $n \mathrm{BA}_{93}-b-\mathrm{NIPAm}_{93}$ on $\mathrm{NaCl}$ aq. at $25^{\circ} \mathrm{C} . \quad A=3.7-4.0 \mathrm{~nm}^{2}$

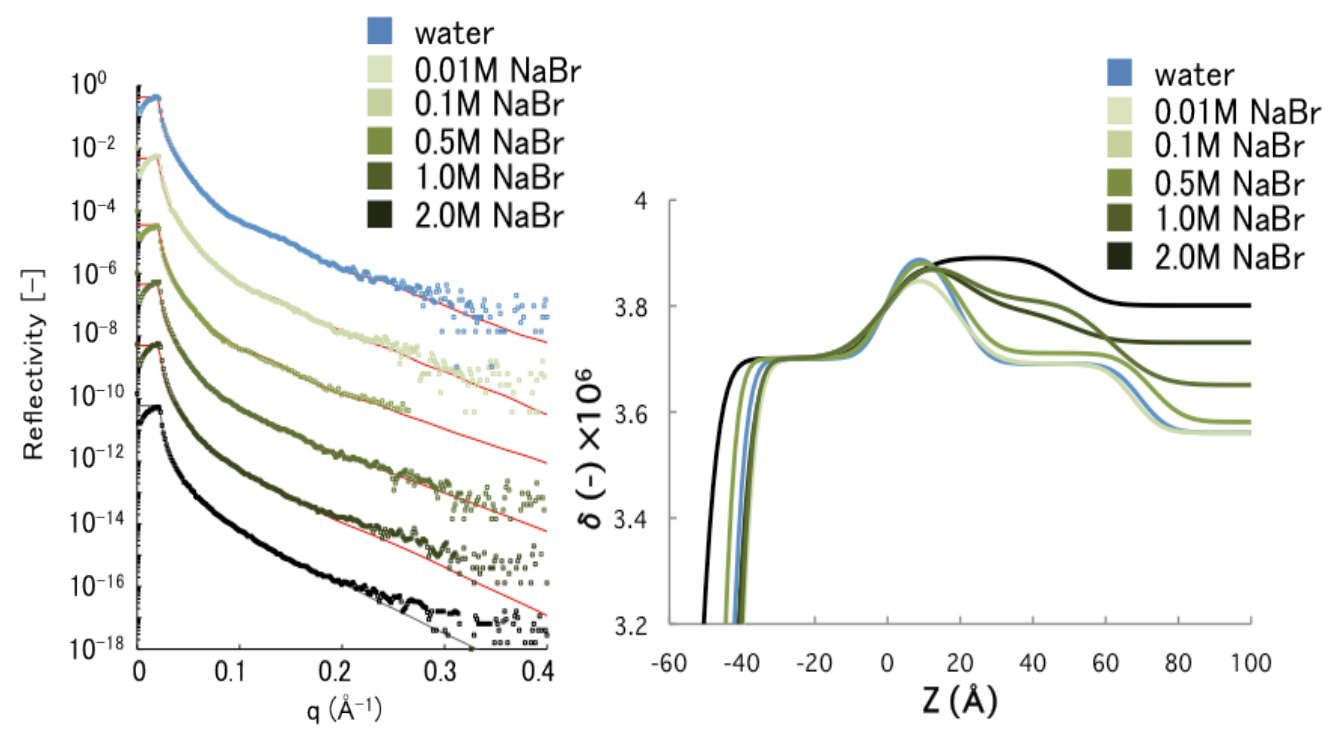

Figure S8 XR profiles (left) and density profiles (right) of $n \mathrm{BA}_{93}-b$-NIPAm 93 monolayer on $\mathrm{NaBr}$ aq. at $25^{\circ} \mathrm{C} . A=3.3$ $\mathrm{nm}^{2}$ 
Table S1. Fitting parameters for $n \mathrm{BA}_{93}-b$-NIPAm 93 monolayer on the water at $25^{\circ} \mathrm{C}$

\begin{tabular}{|c|c|c|c|c|c|c|c|c|c|c|c|c|c|c|c|}
\hline$\pi$ & \multicolumn{3}{|c|}{ hydrophobic layer } & \multicolumn{3}{|c|}{ carpet layer } & \multicolumn{3}{|c|}{ brush layer } & \multicolumn{2}{|c|}{ subphase } & \multirow{2}{*}{$\begin{array}{c}\begin{array}{c}\text { brush } \\
\text { stretch }\end{array} \\
(\%)\end{array}$} & \multirow{2}{*}{$\frac{\text { Area }}{\left(\mathrm{nm}^{2}\right)}$} & \multirow{2}{*}{$\begin{array}{c}\begin{array}{c}\text { brush } \\
\text { density }\end{array} \\
\left(\mathrm{nm}^{-2}\right)\end{array}$} & \multirow{2}{*}{$\mathrm{n}$} \\
\hline$(\mathrm{mN} / \mathrm{m})$ & $\delta$ & $d$ & $\sigma$ & $\delta$ & $d$ & $\sigma$ & $\delta$ & $d$ & $\sigma$ & $\delta$ & $\sigma$ & & & & \\
\hline 28 & \multirow{5}{*}{3.70} & 26 & 4.9 & 3.81 & 29 & 5.0 & - & - & - & \multirow{5}{*}{3.56} & 6.0 & - & 7.2 & 0.14 & - \\
\hline 29 & & 29 & 5.2 & 3.86 & 30 & 8.0 & - & - & - & & 8.0 & - & 6.5 & 0.16 & - \\
\hline 29.5 & & 39 & 4.8 & 3.84 & 22 & 8.0 & 3.66 & 46 & 4.0 & & 9.0 & 48.1 & 4.8 & 0.21 & 12.0 \\
\hline 30 & & 44 & 4.2 & 3.84 & 23 & 8.0 & 3.66 & 59 & 4.0 & & 8.0 & 56.0 & 4.3 & 0.24 & 13.0 \\
\hline 31 & & 49 & 4.3 & 3.84 & 22 & 9.0 & 3.69 & 73 & 3.0 & & 10.0 & 58.1 & 3.8 & 0.26 & 12.2 \\
\hline
\end{tabular}

Table S2. Fitting parameters for $n \mathrm{BA}_{228}-b$-NIPAm ${ }_{76}$ monolayer on the water at $25^{\circ} \mathrm{C}$

\begin{tabular}{|c|c|c|c|c|c|c|c|c|c|c|c|c|c|c|c|}
\hline$\pi$ & \multicolumn{3}{|c|}{ hydrophobic layer } & \multicolumn{3}{|c|}{ carpet layer } & \multicolumn{3}{|c|}{ brush layer } & \multicolumn{2}{|c|}{ subphase } & \multirow{2}{*}{$\begin{array}{c}\text { brush } \\
\text { stretch } \\
(\%)\end{array}$} & \multirow{2}{*}{$\begin{array}{l}\text { Area } \\
\left(\mathrm{nm}^{2}\right)\end{array}$} & \multirow{2}{*}{$\begin{array}{c}\text { brush } \\
\text { density } \\
\left(\mathrm{nm}^{-2}\right)\end{array}$} & \multirow{2}{*}{$\mathrm{n}$} \\
\hline$(\mathrm{mN} / \mathrm{m})$ & $\delta$ & $d$ & $\sigma$ & $\delta$ & $d$ & $\sigma$ & $\delta$ & $d$ & $\sigma$ & $\delta$ & $\sigma$ & & & & \\
\hline 30 & \multirow{5}{*}{3.70} & 69 & 5.0 & 3.77 & 31 & 6.0 & - & - & - & \multirow{5}{*}{3.56} & 4.0 & - & 6.6 & 0.15 & - \\
\hline 30.5 & & 83 & 4.8 & 3.83 & 30 & 4.0 & - & - & - & & 8.0 & - & 5.5 & 0.18 & - \\
\hline 31 & & 104 & 4.8 & 3.84 & 23 & 4.0 & 3.66 & 34 & 4.0 & & 9.0 & 51.9 & 4.4 & 0.23 & 13.5 \\
\hline 32 & & 123 & 4.8 & 3.85 & 24 & 6.0 & 3.67 & 42 & 7.0 & & 9.0 & 53.3 & 3.7 & 0.27 & 10.9 \\
\hline 33 & & 134 & 5.6 & 3.84 & 24 & 9.0 & 3.68 & 51 & 7.0 & & 9.0 & 56.4 & 3.4 & 0.29 & 10.5 \\
\hline
\end{tabular}

Table S3. Fitting parameters for $n \mathrm{BA}_{205}-b-\mathrm{NIPAm}_{29}$ monolayer on the water at $25^{\circ} \mathrm{C}$

\begin{tabular}{|c|c|c|c|c|c|c|c|c|c|c|c|c|c|c|}
\hline$\pi$ & \multicolumn{3}{|c|}{ hydrophobic layer } & \multicolumn{3}{|c|}{ carpet layer } & \multicolumn{3}{|c|}{ brush layer } & \multicolumn{2}{|c|}{ subphase } & \multirow{2}{*}{$\begin{array}{c}\begin{array}{c}\text { brush } \\
\text { stretch }\end{array} \\
(\%)\end{array}$} & \multirow{2}{*}{$\frac{\text { Area }}{\left(\mathrm{nm}^{2}\right)}$} & \multirow{2}{*}{$\begin{array}{c}\begin{array}{c}\text { brush } \\
\text { density }\end{array} \\
\left(\mathrm{nm}^{-2}\right)\end{array}$} \\
\hline$(\mathrm{mN} / \mathrm{m})$ & $\delta$ & $d$ & $\sigma$ & $\delta$ & $d$ & $\sigma$ & $\delta$ & d & $\sigma$ & $\delta$ & $\sigma$ & & & \\
\hline 24 & \multirow{5}{*}{3.70} & 59 & 4.5 & 3.83 & 9 & 4.0 & - & - & - & \multirow{5}{*}{3.56} & 3.0 & - & 7.0 & 0.14 \\
\hline 25 & & 68 & 4.5 & 3.81 & 11 & 4.0 & - & - & - & & 4.0 & - & 6.1 & 0.16 \\
\hline 27 & & 81 & 4.6 & 3.81 & 13 & 4.0 & - & - & - & & 4.0 & - & 5.1 & 0.20 \\
\hline 29 & & 102 & 5.5 & 3.78 & 19 & 7.0 & - & - & - & & 10.0 & - & 4.0 & 0.25 \\
\hline 31 & & 122 & 4.4 & 3.80 & 21 & 8.0 & - & - & - & & 5.0 & - & 3.4 & 0.30 \\
\hline
\end{tabular}

Table S4. Fitting parameters for $n \mathrm{BA}_{93}-b$-NIPAm ${ }_{93}$ monolayer on the water at $4.5 \mathrm{~nm}^{2}$ at $25^{\circ} \mathrm{C}$ and $30^{\circ} \mathrm{C}$

\begin{tabular}{|c|c|c|c|c|c|c|c|c|c|c|c|c|c|c|c|c|}
\hline \multirow[b]{2}{*}{ temp. } & \multicolumn{3}{|c|}{$\begin{array}{c}\text { hydrophobic layer } \\
\text { (nBA) }\end{array}$} & \multicolumn{3}{|c|}{$\begin{array}{c}\text { hydrophobic layer } \\
\text { (NIPAm) }\end{array}$} & \multicolumn{3}{|c|}{ carpet layer } & \multicolumn{3}{|c|}{ brush layer } & \multicolumn{2}{|c|}{ subph } & $\begin{array}{l}\text { brush } \\
\text { stretch }\end{array}$ & \multirow{2}{*}{$n$} \\
\hline & $\delta$ & $d$ & $\sigma$ & $\delta$ & $d$ & $\sigma$ & $\delta$ & d & $\sigma$ & $\delta$ & d & $\sigma$ & $\delta$ & $\sigma$ & $(\%)$ & \\
\hline $25^{\circ} \mathrm{C} 1^{\text {st }}$ & \multirow{4}{*}{3.70} & 42 & 4.4 & - & - & - & 3.85 & 22 & 4.0 & 3.69 & 62 & 5.0 & \multirow{4}{*}{3.56} & 9.0 & 55.8 & 14.8 \\
\hline $30^{\circ} \mathrm{C} 1^{\text {st }}$ & & 42 & 5.8 & 3.90 & 7 & 2.0 & 3.87 & 19 & 4.0 & 3.76 & 20 & 8.0 & & 9.0 & 26.2 & 3.8 \\
\hline $25^{\circ} \mathrm{C} 2^{\text {nd }}$ & & 42 & 5.1 & - & - & - & 3.86 & 22 & 4.0 & 3.66 & 53 & 5.0 & & 12.0 & 48.9 & 12.3 \\
\hline $30^{\circ} \mathrm{C} 2^{\text {nd }}$ & & 42 & 6.8 & 3.90 & 7 & 2.0 & 3.88 & 17 & 3.0 & 3.78 & 21 & 6.0 & & 10.0 & 24.5 & 2.7 \\
\hline
\end{tabular}


Table S5. Fitting parameters for $n \mathrm{BA}_{205}-b-\mathrm{NIPAm}_{29}$ monolayer on the water at $4.3 \mathrm{~nm}^{2}$ at $25^{\circ} \mathrm{C}$ and $30^{\circ} \mathrm{C}$

\begin{tabular}{|c|c|c|c|c|c|c|c|c|c|c|c|c|c|c|c|c|}
\hline \multirow[b]{2}{*}{ temp. } & \multicolumn{3}{|c|}{$\begin{array}{c}\text { hydrophobic layer } \\
\text { (nBA) }\end{array}$} & \multicolumn{3}{|c|}{$\begin{array}{l}\text { hydrophobic layer } \\
\text { (NIPAm) }\end{array}$} & \multicolumn{3}{|c|}{ carpet layer } & \multicolumn{3}{|c|}{ brush layer } & \multicolumn{2}{|c|}{ subphase } & $\begin{array}{l}\text { brush } \\
\text { stretch }\end{array}$ & \multirow{2}{*}{$\mathrm{n}$} \\
\hline & $\delta$ & d & $\sigma$ & $\delta$ & d & $\sigma$ & $\delta$ & $d$ & $\sigma$ & $\delta$ & $d$ & $\sigma$ & $\delta$ & $\sigma$ & $(\%)$ & \\
\hline $25^{\circ} \mathrm{C} 1^{\text {st }}$ & \multirow{4}{*}{3.70} & 106 & 4.7 & - & - & - & 3.85 & 23 & 4.0 & 3.66 & 34 & 7.0 & \multirow{4}{*}{3.56} & 6.0 & 51.2 & 12.9 \\
\hline $30^{\circ} \mathrm{C} 1^{\text {st }}$ & & 106 & 5.6 & 3.90 & 7 & 3.0 & 3.88 & 17 & 3.0 & 3.72 & 15 & 3.0 & & 5.0 & 33.2 & 7.2 \\
\hline $25^{\circ} \mathrm{C} 2^{\text {nd }}$ & & 104 & 3.9 & - & - & - & 3.84 & 23 & 4.0 & 3.66 & 34 & 8.0 & & 8.0 & 51.9 & 13.5 \\
\hline $30^{\circ} \mathrm{C} 2^{\text {nd }}$ & & 106 & 5.9 & 3.90 & 7 & 3.0 & 3.88 & 13 & 2.0 & 3.75 & 16 & 3.0 & & 7.0 & 23.3 & 2.3 \\
\hline
\end{tabular}

Table S6. Fitting parameters for $n \mathrm{BA}_{205}-b$-NIPAm ${ }_{29}$ monolayer on the water at $3.6 \mathrm{~nm}^{2}$ at $25^{\circ} \mathrm{C}$ and $30^{\circ} \mathrm{C}$

\begin{tabular}{|c|c|c|c|c|c|c|c|c|c|c|c|c|c|c|c|}
\hline \multirow[b]{2}{*}{ temp. } & \multicolumn{3}{|c|}{$\begin{array}{c}\text { hydrophobic layer } \\
(n \mathrm{BA})\end{array}$} & \multicolumn{3}{|c|}{$\begin{array}{l}\text { hydrophobic layer } \\
\text { (NIPAm) }\end{array}$} & \multicolumn{3}{|c|}{ carpet layer } & \multicolumn{3}{|c|}{ brush layer } & \multicolumn{2}{|c|}{ subphase } & \multirow{2}{*}{$\begin{array}{c}\text { brush } \\
\text { stretch } \\
(\%)\end{array}$} \\
\hline & $\delta$ & $d$ & $\sigma$ & $\delta$ & $d$ & $\sigma$ & $\delta$ & $d$ & $\sigma$ & $\delta$ & $d$ & $\sigma$ & $\delta$ & $\sigma$ & \\
\hline $25^{\circ} \mathrm{C} 1^{\text {st }}$ & \multirow{4}{*}{3.70} & 112 & 3.8 & - & - & - & 3.79 & 20 & 6.0 & - & - & - & \multirow{4}{*}{3.56} & 3.0 & - \\
\hline $30^{\circ} \mathrm{C} 1^{\text {st }}$ & & 115 & 6.6 & 3.90 & 4 & 2.0 & 3.82 & 13 & 2.0 & - & - & - & & 9.0 & - \\
\hline $25^{\circ} \mathrm{C} 2^{\text {nd }}$ & & 111 & 3.8 & - & - & - & 3.79 & 20 & 6.0 & - & - & - & & 3.0 & - \\
\hline $30^{\circ} \mathrm{C} 2^{\text {nd }}$ & & 116 & 6.3 & 3.90 & 4 & 2.0 & 3.82 & 13 & 2.0 & - & - & - & & 7.0 & - \\
\hline
\end{tabular}

Table S7. Fitting parameters for $n \mathrm{BA}_{93}-b$-NIPAm 93 monolayer on the water at $30^{\circ} \mathrm{C}$

\begin{tabular}{|c|c|c|c|c|c|c|c|c|c|c|c|c|c|c|c|c|}
\hline$\pi$ & \multicolumn{3}{|c|}{$\begin{array}{c}\text { hydrophobic layer } \\
\text { (nBA) }\end{array}$} & \multicolumn{3}{|c|}{$\begin{array}{c}\text { hydrophobic } \\
\text { layer (NIPAm) }\end{array}$} & \multicolumn{3}{|c|}{ carpet layer } & \multicolumn{3}{|c|}{ brush layer } & \multicolumn{2}{|c|}{ subphase } & $\begin{array}{l}\text { brush } \\
\text { stretch }\end{array}$ & $\begin{array}{l}\text { brush } \\
\text { density }\end{array}$ \\
\hline$(\mathrm{mN} / \mathrm{m})$ & $\delta$ & $d$ & $\sigma$ & $\delta$ & d & $\sigma$ & $\delta$ & $d$ & $\sigma$ & $\delta$ & $d$ & $\sigma$ & $\delta$ & $\sigma$ & $(\%)$ & $\left(\mathrm{nm}^{-2}\right)$ \\
\hline 32 & \multirow{4}{*}{3.70} & 30 & 6.9 & 3.90 & 9 & 3.0 & 3.83 & 20 & 2.0 & - & - & - & \multirow{4}{*}{3.56} & 9.0 & - & 0.16 \\
\hline 33 & & 37 & 7.2 & 3.90 & 9 & 3.0 & 3.85 & 25 & 3.0 & - & - & - & & 12.0 & - & 0.19 \\
\hline 33.5 & & 46 & 7.1 & 3.90 & 7 & 3.0 & 3.87 & 23 & 2.0 & 3.75 & 20 & 5.0 & & 8.0 & 30.3 & 0.25 \\
\hline 34 & & 53 & 6.2 & 3.90 & 8 & 3.0 & 3.88 & 22 & 2.0 & 3.77 & 27 & 5.0 & & 6.0 & 30.9 & 0.29 \\
\hline
\end{tabular}

Table S8. Fitting parameters for $n \mathrm{BA}_{228}-b-\mathrm{NIPAm}_{76}$ monolayer on the water at $30^{\circ} \mathrm{C}$

\begin{tabular}{|c|c|c|c|c|c|c|c|c|c|c|c|c|c|c|c|c|}
\hline$\pi$ & \multicolumn{3}{|c|}{$\begin{array}{c}\text { hydrophobic layer } \\
(n \mathrm{BA})\end{array}$} & \multicolumn{3}{|c|}{$\begin{array}{l}\text { hydrophobic } \\
\text { layer (NIPAm) }\end{array}$} & \multicolumn{3}{|c|}{ carpet layer } & \multicolumn{3}{|c|}{ brush layer } & \multicolumn{2}{|c|}{ subphase } & \multirow{2}{*}{$\begin{array}{c}\begin{array}{c}\text { brush } \\
\text { stretch }\end{array} \\
(\%)\end{array}$} & \multirow{2}{*}{$\begin{array}{c}\begin{array}{c}\text { brush } \\
\text { density }\end{array} \\
\left(\mathrm{nm}^{-2}\right)\end{array}$} \\
\hline$(\mathrm{mN} / \mathrm{m})$ & $\delta$ & $d$ & $\sigma$ & $\delta$ & $d$ & $\sigma$ & $\delta$ & $d$ & $\sigma$ & $\delta$ & $d$ & $\sigma$ & $\delta$ & $\sigma$ & & \\
\hline 35 & \multirow{4}{*}{3.70} & 83 & 7.0 & 3.90 & 8 & 3.0 & 3.83 & 21 & 3.0 & - & - & - & \multirow{4}{*}{3.56} & 15.0 & - & 0.18 \\
\hline 35.5 & & 94 & 6.9 & 3.90 & 7 & 4.0 & 3.86 & 22 & 3.0 & - & - & - & & 12.0 & - & 0.20 \\
\hline 36 & & 106 & 6.2 & 3.90 & 7 & 3.0 & 3.88 & 17 & 4.0 & 3.72 & 15 & 8.0 & & 6.0 & 33.2 & 0.23 \\
\hline 37 & & 122 & 5.7 & 3.90 & 9 & 4.0 & 3.85 & 19 & 3.0 & 3.77 & 14 & 3.0 & & 8.0 & 30.4 & 0.27 \\
\hline
\end{tabular}


Table S9. Fitting parameters for $n \mathrm{BA}_{205}-b$-NIPAm 29 monolayer on the water at $30^{\circ} \mathrm{C}$

\begin{tabular}{|c|c|c|c|c|c|c|c|c|c|c|c|c|c|c|c|c|}
\hline & \multicolumn{3}{|c|}{$\begin{array}{c}\text { hydrophobic layer } \\
(n \mathrm{BA})\end{array}$} & \multicolumn{3}{|c|}{$\begin{array}{l}\text { hydrophobic } \\
\text { layer (NIPAm) }\end{array}$} & \multicolumn{3}{|c|}{ carpet layer } & \multicolumn{3}{|c|}{ brush layer } & \multicolumn{2}{|c|}{ subphase } & \multirow{2}{*}{$\begin{array}{c}\begin{array}{c}\text { brush } \\
\text { stretch }\end{array} \\
(\%)\end{array}$} & \multirow{2}{*}{$\begin{array}{r}\begin{array}{r}\text { brush } \\
\text { density }\end{array} \\
\left(\mathrm{nm}^{-2}\right)\end{array}$} \\
\hline$(\mathrm{mN} / \mathrm{m})$ & $\delta$ & $d$ & $\sigma$ & $\delta$ & $d$ & $\sigma$ & $\delta$ & $d$ & $\sigma$ & $\delta$ & d & $\sigma$ & $\delta$ & $\sigma$ & & \\
\hline 25 & \multirow{4}{*}{3.70} & 62 & 6.4 & - & - & - & 3.88 & 8 & 4.0 & - & - & - & \multirow{4}{*}{3.56} & 4.0 & - & 0.15 \\
\hline 27 & & 70 & 6.3 & - & - & - & 3.88 & 9 & 3.0 & - & - & - & & 4.0 & - & 0.17 \\
\hline 29 & & 87 & 7.3 & 3.90 & 5 & 2.0 & 3.84 & 9 & 2.0 & - & - & - & & 5.0 & - & 0.21 \\
\hline 31 & & 104 & 7.0 & 3.90 & 5 & 2.0 & 3.83 & 13 & 2.0 & - & - & - & & 9.0 & - & 0.25 \\
\hline
\end{tabular}

Table S10. Fitting parameters for $n \mathrm{BA}_{93}-b$-NIPAm 93 monolayer on $\mathrm{NaF}$ aq. at $25^{\circ} \mathrm{C}$

\begin{tabular}{|c|c|c|c|c|c|c|c|c|c|c|c|c|c|c|}
\hline Cs & \multicolumn{3}{|c|}{ hydrophobic layer } & \multicolumn{3}{|c|}{ carpet layer } & \multicolumn{3}{|c|}{ brush layer } & \multicolumn{2}{|c|}{ subphase } & \multirow{2}{*}{$\begin{array}{c}\begin{array}{c}\text { brush } \\
\text { stretch }\end{array} \\
(\%)\end{array}$} & \multirow{2}{*}{$\begin{array}{c}\begin{array}{c}\text { brush } \\
\text { density }\end{array} \\
\left(\mathrm{nm}^{-2}\right)\end{array}$} & \multirow{2}{*}{$\mathrm{n}$} \\
\hline (M) & $\delta$ & $d$ & $\sigma$ & $\delta$ & $d$ & $\sigma$ & $\delta$ & $d$ & $\sigma$ & $\delta$ & $\sigma$ & & & \\
\hline 0 & \multirow{4}{*}{3.70} & 46 & 4.9 & 3.85 & 22 & 7.0 & 3.68 & 58 & 5.0 & 3.56 & 7.0 & 48.3 & 0.24 & 10.8 \\
\hline 0.01 & & 48 & 5.1 & 3.87 & 20 & 8.0 & 3.75 & 40 & 8.0 & 3.56 & 7.0 & 31.3 & 0.26 & 4.6 \\
\hline 0.1 & & 51 & 5.3 & 3.88 & 21 & 9.0 & 3.78 & 36 & 7.0 & 3.57 & 12.0 & 26.1 & 0.27 & 3.0 \\
\hline 0.5 & & 55 & 5.7 & 3.89 & 48 & 6.0 & - & - & - & 3.60 & 14.0 & - & 0.29 & 0.0 \\
\hline
\end{tabular}

Table S11. Fitting parameters for $n \mathrm{BA}_{93}-b-\mathrm{NIPAm}_{93}$ monolayer on $\mathrm{NaCl}$ aq. at $25^{\circ} \mathrm{C}$

\begin{tabular}{|c|c|c|c|c|c|c|c|c|c|c|c|c|c|c|}
\hline \multirow{2}{*}{$\frac{\text { Cs }}{(M)}$} & \multicolumn{3}{|c|}{ hydrophobic layer } & \multicolumn{3}{|c|}{ carpet layer } & \multicolumn{3}{|c|}{ brush layer } & \multicolumn{2}{|c|}{ subphase } & \multirow{2}{*}{$\begin{array}{c}\begin{array}{c}\text { brush } \\
\text { stretch }\end{array} \\
\%)\end{array}$} & \multirow{2}{*}{$\begin{array}{c}\begin{array}{c}\text { brush } \\
\text { density }\end{array} \\
\left(\mathrm{nm}^{-2}\right)\end{array}$} & \multirow{2}{*}{$n$} \\
\hline & $\delta$ & $d$ & $\sigma$ & $\delta$ & $d$ & $\sigma$ & $\delta$ & $d$ & $\sigma$ & $\delta$ & $\sigma$ & & & \\
\hline 0 & \multirow{6}{*}{3.70} & 46 & 4.9 & 3.85 & 22 & 7.0 & 3.68 & 58 & 5.0 & 3.56 & 7.0 & 48.3 & 0.24 & 10.8 \\
\hline 0.01 & & 45 & 5.0 & 3.87 & 20 & 7.0 & 3.70 & 50 & 10.0 & 3.56 & 8.0 & 39.8 & 0.24 & 8.2 \\
\hline 0.1 & & 44 & 5.0 & 3.87 & 22 & 9.0 & 3.72 & 35 & 4.0 & 3.57 & 9.0 & 32.4 & 0.23 & 5.9 \\
\hline 0.5 & & 48 & 4.3 & 3.89 & 23 & 8.0 & 3.79 & 30 & 7.0 & 3.61 & 9.0 & 28.5 & 0.26 & 3.6 \\
\hline 1.0 & & 50 & 5.9 & 3.89 & 42 & 10.0 & - & - & - & 3.64 & 9.0 & - & 0.27 & 0.0 \\
\hline 2.0 & & 55 & 5.7 & 3.89 & 48 & 13.0 & - & - & - & 3.72 & 11.0 & - & 0.29 & 0.0 \\
\hline
\end{tabular}


Table S12. Fitting parameters for $n \mathrm{BA}_{93}-b$-NIPAm 93 monolayer on $\mathrm{NaBr}$ aq. at $25^{\circ} \mathrm{C}$

\begin{tabular}{|c|c|c|c|c|c|c|c|c|c|c|c|c|c|c|}
\hline Cs & \multicolumn{3}{|c|}{ hydrophobic layer } & \multicolumn{3}{|c|}{ carpet layer } & \multicolumn{3}{|c|}{ brush layer } & \multicolumn{2}{|c|}{ subphase } & \multirow{2}{*}{$\begin{array}{c}\begin{array}{c}\text { brush } \\
\text { stretch }\end{array} \\
(\%)\end{array}$} & \multirow{2}{*}{$\begin{array}{c}\begin{array}{c}\text { brush } \\
\text { density }\end{array} \\
\left(\mathrm{nm}^{-2}\right)\end{array}$} & \multirow{2}{*}{$\mathrm{n}$} \\
\hline (M) & $\delta$ & $d$ & $\sigma$ & $\delta$ & $d$ & $\sigma$ & $\delta$ & d & $\sigma$ & $\delta$ & $\sigma$ & & & \\
\hline 0 & \multirow{6}{*}{3.70} & 46 & 4.9 & 3.85 & 22 & 7.0 & 3.68 & 58 & 5.0 & 3.56 & 7.0 & 48.3 & 0.24 & 10.8 \\
\hline 0.01 & & 43 & 5.4 & 3.86 & 20 & 9.0 & 3.69 & 51 & 7.0 & 3.56 & 7.0 & 48.2 & 0.23 & 9.7 \\
\hline 0.1 & & 49 & 4.5 & 3.87 & 21 & 6.0 & 3.71 & 52 & 7.0 & 3.58 & 7.0 & 41.3 & 0.26 & 7.5 \\
\hline 0.5 & & 45 & 5.1 & 3.88 & 21 & 8.0 & 3.76 & 37 & 8.0 & 3.65 & 10.0 & 32.2 & 0.24 & 5.5 \\
\hline 1.0 & & 47 & 5.9 & 3.89 & 22 & 7.0 & 3.79 & 29 & 9.0 & 3.73 & 9.0 & 26.8 & 0.25 & 3.3 \\
\hline 2.0 & & 57 & 6.3 & 3.89 & 50 & 9.0 & - & - & - & 3.80 & 10.0 & - & 0.31 & 0.0 \\
\hline
\end{tabular}

Table S13. Fitting parameters for $n \mathrm{BA}_{228}-b-\mathrm{NIPAm}_{76}$ monolayer on $\mathrm{NaF}$ aq. at $25^{\circ} \mathrm{C}$

\begin{tabular}{|c|c|c|c|c|c|c|c|c|c|c|c|c|c|c|}
\hline Cs & \multicolumn{3}{|c|}{ hydrophobic layer } & \multicolumn{3}{|c|}{ carpet layer } & \multicolumn{3}{|c|}{ brush layer } & \multicolumn{2}{|c|}{ subphase } & \multirow{2}{*}{$\begin{array}{c}\text { brush } \\
\text { stretch } \\
\text { (\%) }\end{array}$} & \multirow{2}{*}{$\begin{array}{l}\text { brush } \\
\text { density } \\
\left(\mathrm{nm}^{-2}\right)\end{array}$} & \multirow{2}{*}{$\mathrm{n}$} \\
\hline (M) & $\delta$ & d & $\sigma$ & $\delta$ & d & $\sigma$ & $\delta$ & d & $\sigma$ & $\delta$ & $\sigma$ & & & \\
\hline 0 & \multirow{4}{*}{3.70} & 123 & 4.8 & 3.85 & 24 & 6.0 & 3.67 & 42 & 7.0 & 3.56 & 9.0 & 53.3 & 0.27 & 10.9 \\
\hline 0.01 & & 119 & 4.9 & 3.84 & 22 & 9.0 & 3.68 & 28 & 8.0 & 3.56 & 8.0 & 32.6 & 0.26 & 4.8 \\
\hline 0.1 & & 117 & 5.5 & 3.88 & 21 & 7.0 & 3.78 & 20 & 7.0 & 3.57 & 10.0 & 26.0 & 0.26 & 2.9 \\
\hline 0.5 & & 116 & 6.2 & 3.89 & 34 & 8.0 & - & - & - & 3.60 & 9.0 & - & 0.25 & 0.0 \\
\hline
\end{tabular}

Table S14 Fitting parameters for $n \mathrm{BA}_{228}-b$-NIPAm ${ }_{76}$ monolayer on $\mathrm{NaCl}$ aq. at $25^{\circ} \mathrm{C}$

\begin{tabular}{|c|c|c|c|c|c|c|c|c|c|c|c|c|c|c|}
\hline Cs & \multicolumn{3}{|c|}{ hydrophobic layer } & \multicolumn{3}{|c|}{ carpet layer } & \multicolumn{3}{|c|}{ brush layer } & \multicolumn{2}{|c|}{ subphase } & \multirow{2}{*}{$\begin{array}{c}\begin{array}{c}\text { brush } \\
\text { stretch }\end{array} \\
(\%)\end{array}$} & \multirow{2}{*}{$\begin{array}{c}\begin{array}{c}\text { brush } \\
\text { density }\end{array} \\
\left(\mathrm{nm}^{-2}\right)\end{array}$} & \multirow{2}{*}{$\mathrm{n}$} \\
\hline (M) & $\delta$ & $d$ & $\sigma$ & $\delta$ & $d$ & $\sigma$ & $\delta$ & $d$ & $\sigma$ & $\delta$ & $\sigma$ & & & \\
\hline 0 & \multirow{6}{*}{3.70} & 123 & 4.8 & 3.85 & 24 & 6.0 & 3.67 & 42 & 7.0 & 3.56 & 9.0 & 53.3 & 0.27 & 10.9 \\
\hline 0.01 & & 123 & 5.6 & 3.85 & 22 & 5.0 & 3.74 & 29 & 7.0 & 3.56 & 12.0 & 33.8 & 0.27 & 4.9 \\
\hline 0.1 & & 118 & 6.3 & 3.87 & 23 & 15.0 & 3.72 & 25 & 5.0 & 3.57 & 7.0 & 35.9 & 0.26 & 6.0 \\
\hline 0.5 & & 113 & 5.9 & 3.88 & 21 & 8.0 & 3.80 & 17 & 6.0 & 3.61 & 7.0 & 23.3 & 0.24 & 2.3 \\
\hline 1.0 & & 114 & 6.1 & 3.89 & 33 & 8.0 & - & - & - & 3.64 & 8.0 & - & 0.25 & 0.0 \\
\hline 2.0 & & 118 & 6.3 & 3.89 & 35 & 10.0 & - & - & - & 3.72 & 12.0 & - & 0.26 & 0.0 \\
\hline
\end{tabular}


Table S15. Fitting parameters for $n \mathrm{BA}_{228}-b$-NIPAm ${ }_{76}$ monolayer on $\mathrm{NaBr}$ aq. at $25^{\circ} \mathrm{C}$

\begin{tabular}{|c|c|c|c|c|c|c|c|c|c|c|c|c|c|c|}
\hline Cs & \multicolumn{3}{|c|}{ hydrophobic layer } & \multicolumn{3}{|c|}{ carpet layer } & \multicolumn{3}{|c|}{ brush layer } & \multicolumn{2}{|c|}{ subphase } & \multirow{2}{*}{$\begin{array}{c}\begin{array}{c}\text { brush } \\
\text { stretch }\end{array} \\
(\%)\end{array}$} & \multirow{2}{*}{$\begin{array}{c}\begin{array}{c}\text { brush } \\
\text { density }\end{array} \\
\left(\mathrm{nm}^{-2}\right)\end{array}$} & \multirow{2}{*}{$\mathrm{n}$} \\
\hline (M) & $\delta$ & $d$ & $\sigma$ & $\delta$ & $d$ & $\sigma$ & $\delta$ & $d$ & $\sigma$ & $\delta$ & $\sigma$ & & & \\
\hline 0 & \multirow{6}{*}{3.70} & 123 & 4.8 & 3.85 & 24 & 6.0 & 3.67 & 42 & 7.0 & 3.56 & 9.0 & 53.3 & 0.27 & 10.9 \\
\hline 0.01 & & 118 & 5.8 & 3.85 & 23 & 6.0 & 3.71 & 32 & 7.0 & 3.56 & 9.0 & 40.5 & 0.26 & 7.5 \\
\hline 0.1 & & 119 & 5.4 & 3.86 & 24 & 8.0 & 3.74 & 25 & 6.0 & 3.58 & 7.0 & 35.9 & 0.26 & 6.1 \\
\hline 0.5 & & 124 & 6.0 & 3.88 & 24 & 5.0 & 3.79 & 22 & 6.0 & 3.65 & 9.0 & 30.6 & 0.27 & 3.8 \\
\hline 1.0 & & 119 & 6.2 & 3.89 & 20 & 7.0 & 3.87 & 19 & 7.0 & 3.73 & 9.0 & 23.2 & 0.26 & 1.8 \\
\hline 2.0 & & 121 & 5.9 & 3.89 & 36 & 10.0 & - & - & - & 3.80 & 12.0 & - & 0.26 & 0.0 \\
\hline
\end{tabular}

Table S16. Fitting parameters for $n \mathrm{BA}_{205}-b$-NIPAm 29 monolayer on $\mathrm{NaF}$ aq. at $25^{\circ} \mathrm{C}$

\begin{tabular}{|c|c|c|c|c|c|c|c|c|c|c|c|c|c|}
\hline Cs & \multicolumn{3}{|c|}{ hydrophobic layer } & \multicolumn{3}{|c|}{ carpet layer } & \multicolumn{3}{|c|}{ brush layer } & \multicolumn{2}{|c|}{ subphase } & $\begin{array}{l}\text { brush } \\
\text { stretch }\end{array}$ & $\begin{array}{l}\text { brush } \\
\text { density }\end{array}$ \\
\hline (M) & $\delta$ & d & $\sigma$ & $\delta$ & d & $\sigma$ & $\delta$ & $d$ & $\sigma$ & $\delta$ & $\sigma$ & $(\%)$ & $\left(\mathrm{nm}^{-2}\right)$ \\
\hline 0 & \multirow{4}{*}{3.70} & 122 & 4.4 & 3.80 & 21 & 8.0 & - & - & - & 3.56 & 5.0 & - & 0.29 \\
\hline 0.01 & & 117 & 3.6 & 3.81 & 19 & 5.0 & - & - & - & 3.56 & 7.0 & - & 0.29 \\
\hline 0.1 & & 118 & 3.8 & 3.83 & 18 & 6.0 & - & - & - & 3.57 & 7.0 & - & 0.29 \\
\hline 0.5 & & 125 & 4.6 & 3.83 & 19 & 8.0 & - & - & - & 3.60 & 6.0 & - & 0.30 \\
\hline
\end{tabular}

Table S17. Fitting parameters for $n \mathrm{BA}_{228}-b-\mathrm{NIPAm}_{76}$ monolayer on $\mathrm{NaCl}$ aq. at $25^{\circ} \mathrm{C}$

\begin{tabular}{|c|c|c|c|c|c|c|c|c|c|c|c|c|c|}
\hline Cs & \multicolumn{3}{|c|}{ hydrophobic layer } & \multicolumn{3}{|c|}{ carpet layer } & \multicolumn{3}{|c|}{ brush layer } & \multicolumn{2}{|c|}{ subphase } & \multirow{2}{*}{$\begin{array}{c}\begin{array}{c}\text { brush } \\
\text { stretch }\end{array} \\
(\%)\end{array}$} & \multirow{2}{*}{$\begin{array}{c}\begin{array}{c}\text { brush } \\
\text { density }\end{array} \\
\left(\mathrm{nm}^{-2}\right)\end{array}$} \\
\hline (M) & $\delta$ & $d$ & $\sigma$ & $\delta$ & $d$ & $\sigma$ & $\delta$ & $d$ & $\sigma$ & $\delta$ & $\sigma$ & & \\
\hline 0 & \multirow{6}{*}{3.70} & 122 & 4.4 & 3.80 & 21 & 8.0 & - & - & - & 3.56 & 5.0 & - & 0.29 \\
\hline 0.01 & & 125 & 4.3 & 3.79 & 22 & 6.0 & - & - & - & 3.56 & 5.0 & - & 0.30 \\
\hline 0.1 & & 125 & 5.0 & 3.81 & 20 & 6.0 & - & - & - & 3.57 & 7.0 & - & 0.30 \\
\hline 0.5 & & 125 & 4.5 & 3.82 & 20 & 6.0 & - & - & - & 3.61 & 7.0 & - & 0.31 \\
\hline 1.0 & & 129 & 3.8 & 3.82 & 20 & 6.0 & - & - & - & 3.64 & 7.0 & - & 0.31 \\
\hline 2.0 & & 130 & 3.9 & 3.82 & 20 & 6.0 & - & - & - & 3.72 & 4.0 & - & 0.31 \\
\hline
\end{tabular}


Table S18. Fitting parameters for $n \mathrm{BA}_{205}-b$-NIPAm 29 monolayer on $\mathrm{NaBr}$ aq. at $25^{\circ} \mathrm{C}$

\begin{tabular}{|c|c|c|c|c|c|c|c|c|c|c|c|c|c|}
\hline Cs & \multicolumn{3}{|c|}{ hydrophobic layer } & \multicolumn{3}{|c|}{ carpet layer } & \multicolumn{3}{|c|}{ brush layer } & \multicolumn{2}{|c|}{ subphase } & \multirow{2}{*}{$\begin{array}{c}\begin{array}{c}\text { brush } \\
\text { stretch }\end{array} \\
(\%)\end{array}$} & \multirow{2}{*}{$\begin{array}{c}\begin{array}{r}\text { brush } \\
\text { density }\end{array} \\
\left(\mathrm{nm}^{-2}\right)\end{array}$} \\
\hline (M) & $\delta$ & $d$ & $\sigma$ & $\delta$ & $d$ & $\sigma$ & $\delta$ & $d$ & $\sigma$ & $\delta$ & $\sigma$ & & \\
\hline 0 & \multirow{6}{*}{3.70} & 122 & 4.4 & 3.80 & 21 & 8.0 & - & - & - & 3.56 & 5.0 & - & 0.29 \\
\hline 0.01 & & 119 & 4.1 & 3.81 & 19 & 4.0 & - & - & - & 3.56 & 5.0 & - & 0.29 \\
\hline 0.1 & & 126 & 3.5 & 3.82 & 20 & 6.0 & - & - & - & 3.58 & 7.0 & - & 0.30 \\
\hline 0.5 & & 121 & 4.1 & 3.83 & 20 & 6.0 & - & - & - & 3.65 & 7.0 & - & 0.29 \\
\hline 1.0 & & 122 & 5.6 & 3.86 & 19 & 5.0 & - & - & - & 3.73 & 10.0 & - & 0.29 \\
\hline 2.0 & & 124 & 4.7 & 3.88 & 19 & 6.0 & - & - & - & 3.80 & 9.0 & - & 0.30 \\
\hline
\end{tabular}

\section{Calculation of the number of water molecules in brush layer, $n$.}

(A) Calculation of number of water molecules in carpet layer.

The density inside the carpet layer can be estimated by XR fitting via $\delta$ value. This value should be an average of PNIPAm and water molecules. Since we know both of the densities of bulk PNIPAm and water, volume fraction of each component can be estimated. From the volume for water, we could calculate the number of water molecules.

(B) Calculation of number of water molecules in carpet layer.

The following two methods are available.

Method(1): by density.

Same manner as (A) for carpet layer. Since the average density of the brush layer could be estimated by XR fitting, we could estimate the volume for water, from which the number of water molecules in the brush layer was calculated.

Method (2): by volume

Since the amount of PNIPAm chain in the carpet layer could be estimated by (A), the amount of PNIPAm chain in the brush layer can be calculated. Since we know the brush density and the thicknes of the brush layer, the volume fraction of PNIPAm in the brush layer can be calculated. From this value, the volume for water molecules can be estimated, from which the number of water molecules in the brush layer was calculated.

For example, for 93:93 polymer at brush density of 0.21 (Table S1), the number of water molecules was 11.99 by Method (1), and was 11.94 by Method (2), which shows good 
agreement within $0.5 \%$ error. For brush density of 0.24 , we obtained 12.99 and 12.99 , and for the brush density of 0.26 , we obtained 12.18 and 12.24, by Method (1) and (2), respectively. All of these are in good agreement, so we tabulated the values of 12.0, 13.0, and 12.2 for each brush density in Table S1.

\section{Estimation of molecular weight distribution (Mw/Mn) of P(nBA)-b-PNIPAm block copolymer.}

Generally, GPC experiment for amphiphilic block copolymer is limited because of difficulty of selection of solvent (eluent). However, since we found that $\mathrm{P}(n \mathrm{BA})-b$-PNIPAm block copolymer was apparently dissolved in THF, we tried GPC experiment with THF as an eluent. The results were shown below. We performed two experiments, run 1 and run 2, with the same sample solution. As clearly seen in the figure, the GPC charts for two runs are largely different, which means the non-reproducibility. This might be due to the fact that the block copolymer was not well dissolved in the solvent unfortunately. So, we could not estimate the molecular weight distribution of block copolymer in this study very unfortunately.

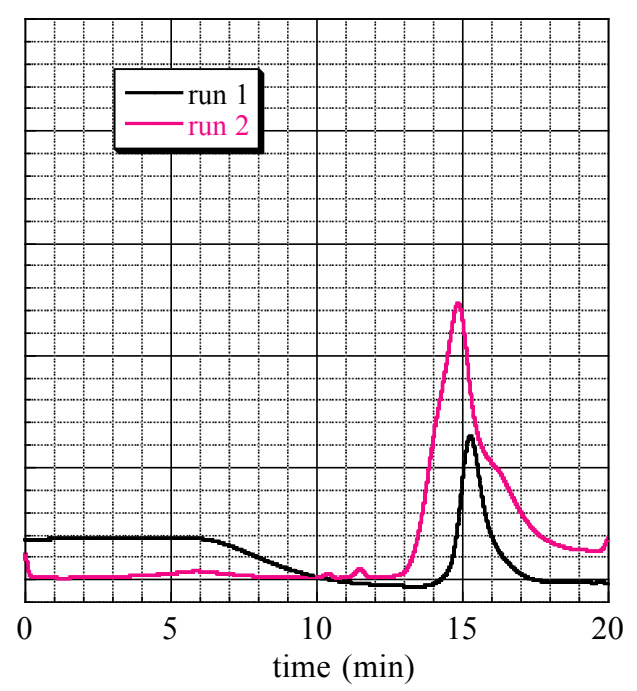

Figure S9 GPC charts for $(n \mathrm{BA})_{93}-b-(\mathrm{NIPAm})_{93}$ with THF as an eluent.

Run 1 gives $\mathrm{Mn}=7110$ and $\mathrm{Mw} / \mathrm{Mn}=1.59$, run 2 gives $\mathrm{Mn}=2730$ and $\mathrm{Mw} / \mathrm{Mn}=5.81$. 